\title{
Atención Conjunta y Desarrollo del Lenguaje Durante el Primer Año de Vida
}

Joint attention and language acquisition during the first year of life

(c) $\frac{10(0)}{\mathrm{BY} N \mathrm{Na}}$

Alejandro Cano Villagrasa Sonia Signes Ribes
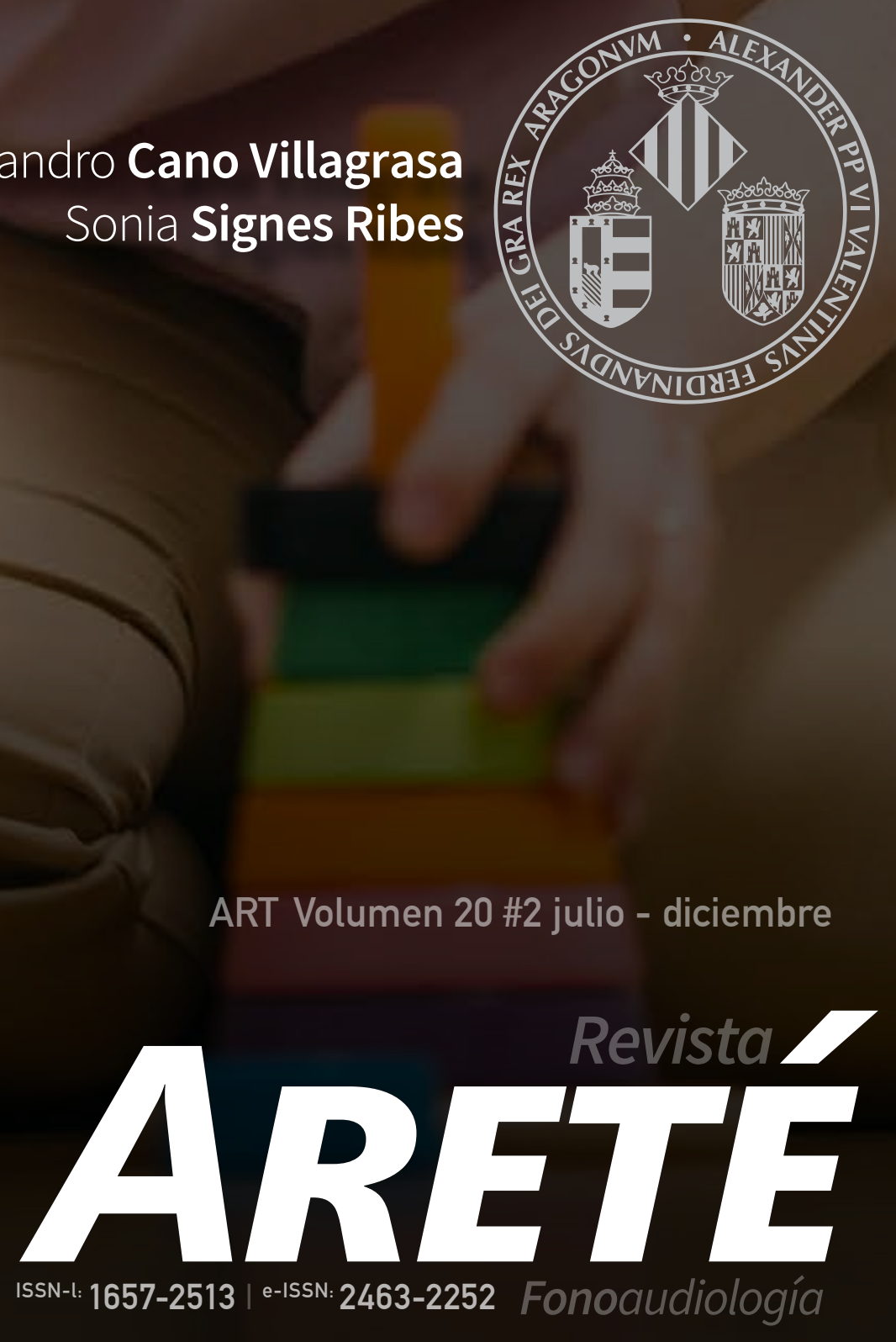
ARETÉ

1657-2513.art.20206

Title: attention and language acquisition during the first year of life

Título: Atención Conjunta y desarrollo del lenguaje durante el primer año de vida

Alt Title / Título alternativo:

[en]: attention and language acquisition during the first year of life[es]: Atención conjunta y desarrollo del lenguaje durante el primer año de vida

Author (s) / Autor (es):

Cano Villagrasa \& Signes Ribes

Keywords / Palabras Clave:

[en]: Communication, language, joint attention, linguistic abilities, language skills, prelinguistics, behavior, development.

[es]: $\quad$ Comunicación, lenguaje, atención conjunta, habilidades lingüísticas, prelingüística, conducta, desarrollo.

Submited: $\quad$ 2020-08-26

Acepted: $\quad$ 2020-11-30

\section{Resumen}

La atención conjunta se centra en el óptimo desarrollo del niño desde el nacimiento hasta el inicio escolar, enfocado en el entorno familiar y social, en los aspectos biopsicosociales. Por otro lado, la adquisición del lenguaje es un proceso complejo que ocurre de forma muy temprana, a pesar de ello es fundamental para el desarrollo humano en

la esfera tanto personal como social. Es por eso por lo que el objetivo que tiene el presente estudio es determinar e identificar la relación

y el papel que juega la atención conjunta en la correcta adquisición del lenguaje y la comunicación en niños de muy corta edad,

utilizando diversas escalas: Early-Social-

Communication Scales y el Inventario de Desarrollo Comunicativo MacArthur. Se estudia mediante la creación de un diseño cuasiexperimental con un solo grupo experimental de 30 niños citados y estudiados en dos etapas diferentes: a los 8 meses (30 sujetos) y a los 12 meses (30 sujetos). Los resultados indican que, si aumentan diversos aspectos de la atención

conjunta, algunos estadios pertenecientes

al lenguaje también se verán incrementados

o mejorados, estableciendo una relación

positiva entre ambas. A lo largo del primer año de vida, estas competencias se ven incrementadas significativamente, preparando los cimientos para la correcta adquisición del lenguaje oral.

\section{Abstract}

The joint attention focuses on the optimal development of the child from birth to the beginning of school, focused on the family and social environment, on biopsychosocial

aspects. On the other hand, language acquisition is a complex process that occurs very early in life, yet in is essential for human development in both the personal and social spheres. That is why the objective of this study is to determine and identify the relationship and the role that early attention plays in the correct acquisition of language and communication in very young children, using various scales: Early-Social-

Communication Scales and MacArthur Communicative Development Inventory. It is studied by creating a longitudinal-transversal

quasi-experimental design, with a single experimental group of 30 children cited and studied in two different stages: at 8 months (30 subjects) and at 12 months (30 subjects). The results indicate that if there were various

aspects of joint attention, some stages pertaining to language will also be increased or improved, establishing a positive relationship between the two. Throughout the first year of life, these competences are significantly increased, preparing the foundations for the correct acquisition of oral language.

\section{Citar como:}

Cano Villagrasa, A. \& Signes Ribes, S. (2020). Atención Conjunta y desarrollo del lenguaje durante el primer año de vida. Areté , 20 (2), 53-61. Obtenido de: https://arete.ibero.edu.co/article/view/ art.20206

Alejandro Cano Villagrasa, PhD MsC BHS

ORCID orcid.org/0000-0001-7330-8987

Source | Filiacion:

Uniiversidad de Valencia

BIO:

Doctorando en Psicología, Logopeda- Universidad de la Laguna-Máster Universitario en Psicología_Universidad de Valencia- Psicopatología Perinatal e Infantil Universidad de Madrid-

City | Ciudad:

España [Esp]
Sonia Signes Ribes, BsH sp

ORCID: orcid.org/0000-0001-9892-8672

Source | Filiacion:

Universidad de Valencia

BIO:

Licenciada en Medicina, investigadora en la Universitat de València, España.

City $\mid$ Ciudad:

España [Esp] 


\section{Atención Conjunta y desarrollo del lenguaje durante el primer año

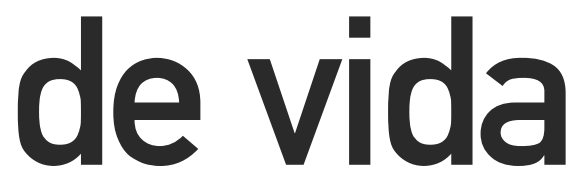

Joint attention and language acquisition during the first year of life Alejandro Cano Villagrasa Sonia Signes Ribes

\section{Introducción}

En la actualidad, múltiples estudios han destacado la importancia de las habilidades cognitivas y sociales, de las cuales emergen la comunicación y el lenguaje, siendo un factor pronóstico clave en su correcta evolución a largo plazo (Caruana, 2015). Entre ellas, se han resaltado diversos factores que entran dentro del desarrollo general del ser humano, siendo la atención conjunta una capacidad básica para establecer intercambios sociales y comunicativos para que pueda desarrollarse el lenguaje (Pfeiffer, 2014)

El lenguaje se puede entender como una herramienta de interacción social que nace de la exposición constante a un ambiente lingüístico (Yoo, 2013) Se refiere a un intercambio de comunicación, constituido por un fenómeno biológico relacional, en donde no solo se transmiten palabras o frases, sino también hay factores no verbales como gestos y conductas que expresan, de forma simultánea, función, contenido y forma. Este proceso es principalmente funcional ya que se emplea como herramienta para suplir las necesidades comunicativas en entornos de carácter social, permitiendo al individuo conocerel mundo que le rodeay adquirirotras habilidades también importantes para su desarrollo físico y cognitivo. Debido a ello, el lenguaje no solo implica aspectos fonológicos y gramaticales, sino que es influenciado por aspectos sociales, culturales y afectivos (Olson, 2011) 


\section{Atención Conjunta y desarrollo del lenguaje durante el primer año de vida}

Antes de que se establezca una comunicación verbal por parte de un individuo, es preciso que se adquieran otras habilidades primordiales que abarcan su desarrollo social, cognitivo y físico, que servirán de línea base de las cuales, si se desarrollan correctamente, surgirá la capacidad de hablar y comprender el lenguaje. Una de las habilidades más importantes es la atención conjunta, que será vital para un desarrollo óptimo, no solo para el lenguaje, sino también para otros aspectos del desarrollo cognitivo del niño en las primeras etapas de la infancia (Mundy, 2009)

El término atención conjunta (JA, por sus siglas es inglés (joint attention)) se define como la capacidad que posee una persona para coordinar la atención con un interlocutor social con respecto a algún objeto o evento (Mundy P. K., 2016) En cuanto a su aparición, comienza a manifestarse antes de los 6 meses de vida y es uno de los principales constructos para que el niño establezca su comunicación y conductas sociales. Para que la JA se dé, es necesario una cierta habilidad para dicha coordinación entre la atención entre el objeto y la persona (Cameron-Faulkner, 2015) . Ha sido dividida en dos componentes principales: la iniciación y la respuesta a los inicios de atención conjunta del interlocutor. Por un lado, la respuesta de la JA (RJA) es la capacidad del niño para seguir la dirección indicada por la mirada, un gesto, o cualquier conducta realizada por la otra persona (Mundy P. , 2016). Por otro, la iniciación a esa respuesta (IJA) será una destreza que se adquiere a continuación de la RJA y que consiste en la utilización del contacto visual junto a gestos deícticos por parte del niño para iniciar la atención coordinada con un interlocutor social.

Pueden existir niveles más simples y complejos de IJA que se presentan durante las distintas etapas del desarrollo del niño. Los más simples corresponderían a un niño que establece el contacto visual mientras manipula el objeto o cambia la mirada entre este y otra persona, representando un desarrollo temprano de la referenciación social. Sin embargo, en el nivel más complejo se encontraría un niño que podría señalar con el dedo ese mismo juguete que muestra otra persona estando presente o no el contacto visual coordinado con el mismo (Cochet, 2016). Por ende, se debería destacar conductas declarativas en los que el niño comparte una experiencia o conciencia de un objeto o evento, ya que estas acciones ponen de manifiesto que los niños captan a otras personas como agentes intencionales, puesto que comprenden que esas personas presentan estados mentales internos tales como atención, intereses, deseos e interacciones y que estos pueden ser compartidos y actuar sobre ellos.

Durante estos episodios de interacción, los adultos adaptan su lenguaje y su comunicación a los diferentes focos de interés de los niños. De esta forma, la atención conjunta ayuda notablemente a que los bebés identifiquen el elemento que el adulto está nombrando (Matthews, 2012). El gesto de señalar resulta también determinante en el desarrollo simbólico y en el aprendizaje del lenguaje verbal, ya que mediante este gesto el adulto acota el referente de una manera muy precisa. En este sentido, se ha demostrado una fuerte relación de los comportamientos de atención conjunta con la comunicación verbal posterior, ya que es uno de los factores fundamentales para que se desarrolle correctamente el lenguaje. De forma más concreta, (Kristen, 2011) han demostrado que la capacidad de atención conjunta a los 12 meses de edad predice el vocabulario.

Otro factor indispensable, en este caso cognitivo, para la adquisición del lenguaje es la capacidad del niño para atender de forma conjunta a que está atendiendo su interlocutor, puesto que no solo facilita la adquisición del vocabulario, sino también otros aspectos pragmáticos, sintácticos y fonológicos. Autores como (Bruner, 1983), sostenían que era necesario estudiar de manera detallada todo el sistema de comunicación previo para poder entender la posterior adquisición del lenguaje, dejando otros aspectos cognitivos del niño. Sin embargo, (Butterworth, 1991) había destacado la importante función comunicativa de la atención durante el período prelingüístico, debido a que proporciona información básica sobre los objetos con los que el niño interactúa.

No obstante, es importante puntualizar que el efecto facilitador de la atención conjunta puede verse influido por una variable fundamental en relación con el primer lenguaje, como es el estilo de los padres cuando interactúan con sus hijos. Se ha demostrado que, si la madre sigue el foco de atención del niño cuando nombra los objetos que su hijo observa, existe una relación significativa y positiva con el desarrollo del léxico, pero si, por el contrario, el cuidador tiene un estilo basado en modificar constantemente el foco atencional del niño, y nombra objetos a los que el niño no está atendiendo, afecta negativamente al desarrollo del lenguaje. La sensibilidad de los padres se manifiesta en conductas como su habilidad para mantener la atención y la motivación de su hijo hacia la tarea, la cantidad de tiempo dedicada a compartir la atención, así como la capacidad para simplificar la tarea, demostrar y señalar las características relevantes de la misma, y acomodarse a los estados emocionales de los niños (Sodian, 2015) Dichas variables han resultado buenos predictores de la posterior adquisición del vocabulario por el niño.

Las alteraciones que afectan a la atención conjunta y la orientación social son los que distinguen a los niños con alguna patología o retraso en el desarrollo de aquellos que lo hacen con normalidad (Cano Villagrasa, 2020). El desarrollo de la atención conjunta es una coordinación previa fundamental de gran parte de la adquisición temprana del lenguaje y la JA parece ser un pronóstico fiable de la habilidad lingüística concurrente (Ramenzoni, 2016) . Aunque no sea definitiva esta relación entre la no atención conjunta y un posible retraso en los diferentes estadios del lenguaje, es muy probable que surja, y poder detectarla a tiempo resultará vital para remediarla en los periodos críticos de adquisición del lenguaje y la comunicación del individuo (Sorondo, 2015)

Otras alteraciones existentes que puedan influir en el correcto desarrollo del lenguaje infantil es la prematuridad. Debido a la falta de evidencia científica y a estudios que corroboren la correlación entre retraso del lenguaje y prematuridad, habría que señalar que esta condición no garantiza la aparición de un trastorno de lenguaje, ya que los niños no nacidos a término no siempre tienen patologías y mayoritariamente se desarrollan sin ningún tipo de limitación o contratiempo, relacionados sobre todo con el estadio de la pragmática del lenguaje (Kuhn, 2014)

En general, la teoría pragmática del lenguaje se centra en el uso de este y pone una gran importancia en la riqueza del contexto en donde se desarrolla el bebé, adquiriendo relevancia como factor que facilita la comprensión acerca de la adecuación de un acto comunicativo (Acosta, 2013) Con este término se hará referencia a la intención comunicativa con el que el hablante emitirá sus necesidades y deseos. Se distingue, pues, la funcional de las emisiones, así como el significado de esas emisiones transmitidas y, por último, se establecerá al hablante competente como aquel capaz de construir emisiones con intención comunicativa. En esta línea, conviene destacar las investigaciones de (Acosta V. D., 2016). Estos autores se centran en el análisis de dos funciones: las declarativas y las imperativas. Según estos autores, la función protodeclarativa se define como un esfuerzo preverbal que el niño realiza para dirigir la atención del adulto hacia un objeto o evento de su interés. Por otro lado, la función protoimperativa se definiría como el modo, también preverbal, a través del cual el niño intenta que el adulto realice una determinada acción. Todo lo mencionado cobra una gran importancia en cuanto a la evaluación del niño, ya que son 
de los aspectos más importantes para ver si existe alguna patología en el niño de corta edad, puesto que múltiples pruebas evaluativas, así como protocolos diagnósticos se basan en este estadio del lenguaje para comprobar si existe un trastorno en el individuo.

Además de ello, como se ha descrito anteriormente, varios estudios corroboran que la inexistencia de conductas de atención conjunta, serán precursores y signos de alarma a la hora de demostrar este tipo de patología lingüística. Un contratiempo en el correcto desarrollo infantil podría originaría un retraso no solo de estas competencias lingüísticas, sino también de la capacidad para atender de forma conjunta (Laurence B, 2014). Por tanto, si se reúnen estos factores, se concreta que el principal estadio del lenguaje que se ve afectado y manifiesta una posible limitación para el individuo será la pragmática, debido a que aparecerá un retraso del lenguaje y en la producción oral de un niño sobre los 12 meses de edad, en donde tendría que comenzar a articular sus primeras palabras (Maldonado, 2013)

En conclusión, este estudio tiene como objetivo principal determinar e identificar el papel que juega la atención conjunta en la correcta adquisición del lenguaje y la comunicación en niños durante su primera infancia.

\section{Metodología}

Se trata de un estudio cuasi-experimental longitudinal-transversal, con un solo grupo experimental, utilizando para el análisis de los resultados, el estadístico no paramétrico de correlación de Rho de Spearman, debido a la limitada muestra que presenta la investigación. Se pretende comparar, de forma intragrupal, los resultados de las pruebas en las diferentes fases de evaluación a las edades establecidas: 8 y 12 meses. La edad de 12 meses es un periodo crítico en donde se resaltan diferentes habilidades y competencias del desarrollo infantil. Es un momento en donde el apartado motor, lingüístico y social emulsiona y se pueden observar las primeras etapas de estas dimensiones. Por ende, la elección de estos momentos del desarrollo permite realizar una comparación entre un punto de partida en donde los sujetos presentan unas competencias similares (8 meses) y otro en donde emergen los primeros rasgos evolutivos significativos de algunas competencias del desarrollo general infantil: lenguaje, motricidad y sociabilidad (12 meses).

\section{Muestra}

Se han seleccionado un total de 30 niños (19 niñas y 11 niños) de los cuales $48.4 \%$ eran prematuros y $51.6 \%$ nacidos a término; junto a su progenitor (generalmente la madre de media de edad 35 años, 96.77\%). Respecto a estos niños, el $48.4 \%$ eran prematuros (sin patologías asociadas, que acudieron previamente a terapias de atención y estimulación temprana, teniendo un seguimiento exhaustivo y presentando un rendimiento similar a los demás niños incluidos en el grupo muestral de esta investigación) y el 51.6\% nacidos a término. En cuanto a la estructuración familiar, el 40\% eran primogénitos, el 53\% tenían 1 hermano y el 6.6\% entre 2 y 3 hermanos. El 93.5\% viven en una estructura familia clásica y el $6.5 \%$ en familias reconstituidas, el $64.5 \%$ viven en un entorno urbano, el $22,6 \%$ en una zona residencial y el $12,9 \%$ en entorno rural. Por último, los ingresos familiares anuales que presentaban las familias fueron: el 3,3\% de 6.000 a $11.999 €, 26,7 \%$ entre 12.000 y $23.999 €$, el $43,3 \%$ entre 24.000 y $35.999 €$, el $23.3 \%$ entre 36.000 y $50.000 €$ y el 3,3\% más de $50.000 €$.
En cuanto a los progenitores, la edad media de las madres fue 35,65 años y de los padres 36,97 años. El 71\% estaban casados, 25,8\% vivían en pareja y el 3,2\% era familia monoparental. Respecto al nivel de estudios que presentaban, por un lado, de las madres el 9,7\% tenía estudios básicos, el 25,8\% medios, es decir, estudios de bachillerato o ciclos formativos, y 64,5\% superiores, correspondientes a los universitarios. Por otro lado, de los padres el 3,3\% no tenía estudios, el 10\% tenía estudios básicos, el 30 \% medios y el 56,7\% superiores. Por último, lo que corresponde a la ocupación laboral, el 32\% de las madres no trabajaba, el 16,1 \% lo hacía a tiempo parcial, el 48,4\% a tiempo completo y el 3,2\% eran pensionistas, y respecto a los padres: el 6,7\% no trabajaba, $10 \%$ trabajaba a tiempo parcial y el $83,35 \%$ lo hacía a tiempo completo.

\section{Criterios de inclusión}

Las familias que se incluyen son las primeras evaluadas para el estudio citado, contándose con datos de la primera evaluación (8 meses) para 30 casos, y una segunda cuando habían alcanzado el primer año de vida en el momento del análisis de los datos del presente estudio, por lo que se incluyeron como un subgrupo.

\section{Criterios de exclusión}

Los criterios principales que excluirán a los sujetos para la realización de este estudio han sido los siguientes: presentar un déficit sensorial, tener menos de 36 semanas de gestación y, por último, presentar una enfermedad motora o cognitiva que impida la correcta realización del estudio.

\section{Instrumentos de recolección de datos y medición}

Para la observación semiestructurada de conductas comunicativas en niños pre-verbales se utiliza el Early Social-Communication Scales (Mundy P. D., 2003) (el cual presenta un diseño semiestructurado de 15 a 25 minutos de duración. Se trata de un instrumento de observación conductual que debe ser registrado en video para su posterior codificación. Se toma especial interés en los parámetros: conductas de atención conjunta (joint attention) que corresponde con el uso de conducta no verbal para compartir con el examinador interés hacia a un juguete o un juego, conductas de demanda (behavioural request) que hacen referencia al uso de conductas no verbales de petición de ayuda al adulto para alcanzar un objeto o conseguir alguna finalidad, y, por último, la interacción social (social interaction), la cual se define como la capacidad del niño de participar en interacciones lúdicas, de afecto positivo en alternancia de turnos con el examinador. Para comprobar la fiabilidad de los datos se codifican los datos recogidos y se utiliza el Índice de Correlación Interclase (ICC) en aquellas variables de conducta de aparición frecuente.

Mediante Inventarios de Desarrollo Comunicativo MacArthur (IDC; (López-Ornat, 2005) se pretende evaluar el proceso de adquisición temprana del lenguaje mediante un conjunto de diferentes manifestaciones: Gestos prelingüísticos, vocalizaciones prelingüísticas, vocabulario y gramática. Se hace uso del primer inventario: Vocalizaciones, primeras palabras y gestos (8 a 15 meses), el cual se divide en tres subescalas que recogen diferentes aspectos del lenguaje verbal y no verbal: Vocalizaciones prelingüisticas, primeras palabras, gestos y acciones.

Por último, se ideó una entrevista semi-estructurada con un total de 17 preguntas abiertas en donde se registraban todos los datos 


\section{Atención Conjunta y desarrollo del lenguaje durante el primer año de vida}

sociodemográficos del núcleo familiar de los sujetos que conforman la muestra de este estudio. Se han incluido variables como: datos personales, profesión y nivel de estudios de los progenitores, número de hermanos, patologías asociadas, estructura familiar, ingresos anuales y otras observaciones de interés.

\section{Procedimiento}

Para la realización de este estudio, se ha pedido el reconocimiento del Comité de Ética, siendo aprobado por este y obteniendo el siguiente número de registro: 2017/0168. Los procedimientos llevados a cabo durante el estudio se resumen en las siguientes fases:

\section{Primera fase o selección de muestra}

En esta primera fase, se realizó una búsqueda de la muestra en diferentes centros médicos y hospitales de la Comunidad Valenciana, que reuniesen las condiciones y características necesarias para la aplicación del estudio; seguido de la selección de aquellos que cumplen los criterios de inclusión previamente establecidos.

Por último, se establece una reunión con los padres para informarles de las sesiones y el procedimiento a seguir, además de concertar las citas para la evaluación de los sujetos según cumplan los 8 y 12 meses de edad.

\section{Segunda fase o Evaluación de la atención conjunta y lenguaje}

Se lleva a cabo la evaluación de la atención conjunta y el lenguaje de los sujetos del estudio en los meses establecidos. En caso de algún contratiempo que perjudique la asistencia en el desarrollo de las sesiones, se propuso otra sesión lo antes posible para que fuera en esa misma franja de edad.

La actividad se realizó en un laboratorio sin ningún estímulo visual que pueda entorpecer o perjudicar al correcto desarrollo de la sesión de evaluación del sujeto en cuestión, estando solo disponible aquellas herramientas y utensilios que se requieren para la prueba. Todas las sesiones son grabadas para su posterior codificación de las conductas observadas, por lo que previamente se obtiene tanto el consentimien- to informado de participación en el estudio como el permiso para realizar dichas grabaciones.

Las sesiones de intervención tuvieron una duración máxima aproximada de 90 minutos y se dividen en las siguientes partes:

Periodo de juego libre: durante este periodo de 8 minutos, el familiar y el niño juegan sin recibir orientación alguna. Se dispone de una caja llena de juguetes de diferentes tipos, colores y tamaños para jugar.

Periodo de evaluación con ESCS: durante este periodo, se realizó el pase de la prueba ESCS, la cual valora la atención conjunta y la interacción del niño con el evaluador. El tiempo establecido para esta parte fue de 20 minutos como máximo.

Periodo de evaluación del lenguaje: en este periodo se efectuó el cuestionario MacArthur, el cual evalúa ciertos aspectos del lenguaje que posee el niño en ese momento de su desarrollo. Este periodo no tiene un tiempo de realización concreto, pero es necesario que no exceda de más de una hora, debido al cansancio que pueda generar las anteriores actividades sumadas a ésta.

\section{Tercera fase o Análisis de resultados obtenidos}

Tras la obtención de datos se procede a codificar las conductas recogidas en la prueba ESCS. Seguidamente, mediante el software informático SPSS versión 23.0, se realizaron los análisis estadísticos pertinentes (análisis descriptivos y correlaciones) y su interpretación.

\section{Resultados}

\section{Atención conjunta y comunicación temprana (ESCS) en 8 y 12 meses}

Las siguientes tablas muestran los estadísticos descriptivos de las variables que evalúan a través del Early Social-Communication Scales (ESCS; (Mundy P. D., 2003), tanto en 8 como en 12 meses de edad del bebé:

Tabla 1.Estadísticos descriptivos de ESCS para niños de 8 y 12 meses

\begin{tabular}{|c|c|c|c|c|}
\hline & \multicolumn{4}{|c|}{ Estadísticos descriptivos ESCS } \\
\hline & \multicolumn{2}{|c|}{8 meses $(n=30)$} & \multicolumn{2}{|c|}{12 meses $(n=30)$} \\
\hline & Media & $\begin{array}{l}\text { Desviación } \\
\text { estándar }\end{array}$ & Media & $\begin{array}{l}\text { Desviación } \\
\text { estándar }\end{array}$ \\
\hline IJA Low & 14,1 & 8,68 & 13,45 & 6,71 \\
\hline IJA High & 0,13 & 0,57 & 6,72 & 3,84 \\
\hline RJA Low & 3,93 & 2,31 & 4,9 & 2,34 \\
\hline RJA High & 0,76 & 1,16 & 5,09 & 2,34 \\
\hline IBR Low & 7,96 & 4,19 & 9,09 & 4,9 \\
\hline IBR High & 0 & 0 & 6,54 & 6,18 \\
\hline
\end{tabular}




\begin{tabular}{ccc|c|c} 
& \multicolumn{4}{c}{ Estadísticos descriptivos ESCS } \\
\cline { 2 - 5 } & Media & $\begin{array}{c}\text { Desviación } \\
\text { estándar }\end{array}$ & Media & $\begin{array}{r}\text { Desviación } \\
\text { estándar }\end{array}$ \\
\hline RBR Low & 0 & 0 & 0 & 0 \\
RBR High & 0 & 0 & 0 & 0 \\
\hline ISITT & 0,86 & 2,83 & 3,36 & 3,85 \\
ISI Low & 0 & 0 & 0 & 0 \\
\hline ISI High & 0 & 0 & 0 & 0 \\
\hline RISI & 4,23 & 1,69 & 5,54 & 1,21 \\
RISITT & 0,23 & 0,62 & 9,9 & 4,67 \\
RISIRI & 0,03 & 0,18 & 1,36 & 1,28 \\
\hline
\end{tabular}

IJA, Imitating joint attention; RJA, Responding to joint attention; IBR, initating behavioural request; RBR, responding to behavioural request; ISI, initating social interaction; RISI, responding to social interaction

A pesar de la diferencia del tamaño de los grupos, se puede apreciar que las conductas que recoge este cuestionario, que reflejan las habilidades de comunicación temprana, se ven incrementadas con la edad, tal y como cabría esperar en bebés que no presentan problemas en el desarrollo, donde se puede ver un mayor incremento en las versiones más complejas (high) de las conductas descritas. Así, existen valores mayores en todas las variables para el grupo de mayor edad. Para conocer si el presentar inicio temprano de estas conductas en 8 meses, favorece una mejor competencia en las mismas a los 12 meses, se realizaron correlaciones de Spearman entre estas dos edades.

Con este estadístico, en el apartado de Inicio y Respuesta de Atención Conjunta, se obtiene que las conductas de IJA Low a los 8 meses van a favorecer la producción de mayor RJA Low también a los 8 meses $(r=0,536(\operatorname{sig}=0,002))$. Además de ello, se encuentra que a cuantas más RJA High haga el niño a los 8 meses, mayores producciones de IJA tendrá a los 12 ( $r=0,627$ (sig=0,039). Por último, se obtuvo que, si el niño realiza muchas IJA Low a los 12 meses, mostrará más RJA High en esa misma edad $(r=0,647$ (sig=0,032).

En cuanto al apartado de Inicio y Respuesta de Conducta, se muestra que a cuantas más conductas de IBR Low realice un niño en 8 meses, más IBR High producirá a los 12 ( $r=0,613$ (sig=0,045). Lo que se traduce en que, si un niño es capaz de realizar conductas como, por ejemplo, mirar a otro individuo cuando el bebé se encuentra manipu- lando el objeto, mejorará la emisión de diversas acciones más complejas a los 12 meses, como mostrar el objeto a otra persona, entre otras.

Por último, referido al apartado de Respuesta de Interacción Social, se encuentra que si el bebé emite más RISIT a los 8 meses, mayores RISI se presentarán en el niño en esa misma edad ( $r=0,418$ (sig=0,022), es decir, si se le proporciona un accesorio como, por ejemplo, un peine o un sombrero con el objetivo de que lo explore y, a continuación, se le invita a que interactúe con un adulto con ese mismo objeto, el niño responderá adecuadamente a esta situación e interactuará de forma conjunta en la edad de 8 meses.

\section{Destreza comunicativa y habilidades lingüísticas (MacArthur) en 8 y 12 meses}

A continuación, se exponen los estadísticos descriptivos de las variables correspondientes a las habilidades prelingüísticas y comunicativas que se evalúan a través del Inventarios de Desarrollo Comunicativo MacArthur (López-Ornat, 2005) tanto en 8 como en 12 meses de edad del bebé:

Tabla 2. Estadísticos descriptivos de Inventario de Desarrollo Comunicativo MacArthur para niños de 8 y 12 meses

\begin{tabular}{|c|c|c|c|c|}
\hline & \multicolumn{4}{|c|}{ Estadísticos descriptivos MacArthur } \\
\hline & \multicolumn{2}{|c|}{8 meses $(n=30)$} & \multicolumn{2}{|c|}{12 meses $(n=30)$} \\
\hline & Media & $\begin{array}{c}\text { Desviación } \\
\text { estándar }\end{array}$ & Media & $\begin{array}{l}\text { Desviación } \\
\text { estándar }\end{array}$ \\
\hline Vocalizaciones & 6,3 & 1,78 & 9,81 & 1,4 \\
\hline Primeras Palabras & 25,26 & 9,88 & 108,36 & 9,78 \\
\hline Acciones y Gestos & 6,33 & 3,17 & 17,36 & 5,51 \\
\hline
\end{tabular}




\section{Atención Conjunta y desarrollo del lenguaje durante el primer año de vida}

Como se puede observar en la tabla 2, las conductas registradas en este instrumento, que corresponden con las destrezas comunicativas y las habilidades lingüísticas del niño, sufren cambios en las medias de cada una de las subescalas de este inventario desde la primera medición a los 8 meses respecto a la siguiente, en los 12 meses de edad. Se aprecia como las medias en los 8 meses son inferiores a las obtenidas a los 12, indicando de esta manera, que los niños mejoran en los diferentes aspectos del lenguaje y comunicación a medida que crecen, por lo que los datos que arroja dicha tabla son los esperados para con los sujetos del estudio. Con el fin de analizar estadísticamente dicha diferencia en las medidas, se expone a continuación las correlaciones de Rho de Spearman correspondientes a estas variables.

Realizando el estadístico mencionado, se obtiene una correlación positiva entre las vocalizaciones y las primeras palabras a los 8 meses $(r=0,457$ (sig=0,011), por lo que, si un niño presenta muchas vocalizaciones, mostrará una mejor comprensión, tanto de palabras como de frases, además de producir emisiones de mayor calidad a los 8 meses. Estas primeras palabras a los 8 meses muestran también dicha correlación con las acciones y gestos en esa edad ( $r=0,377$ ( ig=0,040), lo que se traduce en que la comprensión de palabras y frases, a la vez que presentar emisiones en forma de balbuceo, favorecerán las conductas de juego y gestos que acompañan a la comunicación del bebé en los 8 meses de edad. Por último, además de lo mencionado, se aprecia relación entre las primeras palabras y las acciones y gestos a los 12 meses $(r=0,716$ (sig=0,013).

\section{Relación entre conductas}

\section{comunicativas en 8 y 12 meses}

En este apartado, se presentarán las correlaciones pertinentes entre cada uno de los instrumentos empleados en el estudio con el interés de comparar los diferentes contextos empleados durante la investigación, es decir, en un entorno controlado y en uno natural.

En primer lugar, se encuentra una relación significativa entre las variables de vocalización (MacArthur) e IJA Low en niños de 8 meses $(r=0,413$ (sig=0,023), por lo que, si el niño es capaz de producir emisiones simples de vocales o sílabas, mejorará la interacción en inicio de atención conjunta con un adulto. En segundo lugar, se aprecia que si, en los 12 meses, el niño que muestra una buena señalización durante su juego en un contexto natural (MacArthur), se producirán más RJA High en un entorno controlado en esa misma edad, obteniéndose, de esta manera, datos significativos $(r=0,653$ (sig=0,029). Por último, en tercer lugar, se obtiene que, a los 12 meses, la existencia de vocalizaciones en el niño (MacArthur), producirá un mejor lenguaje expresivo $(r=0,784$ (sig=0,045).

\section{Discusión}

En este estudio, se pretendía comprobar cuál es el papel de la atención conjunta y habilidades de comunicación temprana en el desarrollo lingüístico durante el primer año de vida. Así, se persigue demostrar como la presencia de habilidades de atención conjunta en edades tempranas es una herramienta que ayudaría posteriormente a la correcta adquisición del lenguaje, además de ser un factor de protección ante alguna posible patología referente al lenguaje y a la comunicación.

Como se puede observar, los resultados muestran un nivel de significación en las variables tanto de lenguaje y comunicación, como de atención conjunta, en un contexto controlado. Los niños presentan un cambio en sus medias desde sus 8 meses hasta los 12, lo que implica que, en las puntuaciones en prácticamente todas las variables del estudio, los sujetos consiguen mejorar significativamente debido a su desarrollo madurativo, como cabría esperar. A parte de lo mencionado, se puede apreciar como tanto los diferentes estadios de JA como los de lenguaje y comunicación, mejoran a la par según la edad del niño, siempre y cuando este no presente ninguna patología que impida el correcto desarrollo madurativo. En cuanto a la relación entre las conductas de JA y comunicación en los 8 y 12 años, se resalta una correlación significativa entre varias de ellas, indicando una posible tendencia positiva sobre todo en las vocalizaciones y en el inicio y respuesta de la JA. Estos resultados se traducen en que los niños que comienzan a emitir palabras a los 8 meses podrían presentar una mayor iniciación y respuesta en las conductas de atención conjunta a los 12 meses, favoreciendo el correcto desarrollo y adquisición del lenguaje expresivo y receptivo, estableciéndose como uno de los factores de protección más importantes en la infancia.

Los datos obtenidos en esta investigación son similares a los mostrados en otros estudios como el de (Cameron-Faulkner, 2015), en donde obtienen que los niños de 11 meses comienzan a realizar conductas como el señalado, contacto ocular o alternancia entre el objeto y el adulto de manera notoria. Esto se relaciona significativamente con la producción de lenguaje y comunicación en niños de corta edad, puesto que la atención conjunta, potencia la iniciación de aspectos de la comunicación social, sobre todo del niño respecto al adulto. El uso de estos gestos indica que la interacción con el entorno donde se desarrolla el niño es estimulante por lo que invita y provoca que la comunicación aparezca de manera satisfactoria y óptima de manera natural.

Otra relación aparece en el estudio de (Elgier, 2017), donde se pretendía estudiar la modulación que ejerce el nivel socioeconómico en las primeras habilidades comunicativas del niño. Según sus resultados, se puede afirmar que existe una relación entre la biología, en la base genética ya constituida, interaccionando con el entorno, resultando en el desarrollo de habilidades. Otra relevancia para comentar es que las capacidades de la atención conjunta no implican capacidades de lenguaje oral, juego de ficción y habilidades mentalistas, en donde ya es necesario el manejo consciente de recursos simbólicos y de control metacognitivo. Sugieren que lo que más influye en los episodios de atención conjunta son los métodos estratégicos y otras conductas que de forma común son usadas por los cuidadores y la gente del entorno, afectando tanto a su cualidad y duración, como la capacidad didáctica del episodio de atención conjunta.

Otro estudio que guarda relación es el de (Gutiérrez-Ruiz, 2019) cuyo objetivo fue el de describir los antecedentes del desarrollo y características tempranas. Se obtuvo que los niños sin ningún tipo de patología del desarrollo mejoran sus conductas de atención conjunta a partir de los 12 meses de edad. Además de ello, a esta edad los niños presentan gestos como el señalado o la alternancia entre el objeto y el adulto en momentos de juego con una mayor frecuencia, lo que les facilita la adquisición de nuevas palabras o desarrollar nuevos patrones comunicativos en los cuales, la estimulación en el ambiente doméstico provocará la aparición del lenguaje y la comunicación de forma adecuada.

Los resultados reflejados podrían ser de gran utilidad como una primera aproximación al campo del desarrollo del lenguaje en la infancia, para proceder posteriormente a profundizarse en el mismo. La importancia de esta investigación radica en provocar concientización en las políticas públicas y en la población de la estimulación temprana orientada hacia la comunicación preverbal. Los niños precisan recibir estímulos de forma continua y diaria, desde el momento de su naci- 
miento. Si recibe estímulos pobres, de una forma irregular o en cantidad insuficiente, el cerebro no desarrolla adecuadamente sus capacidades al ritmo y con la calidad necesaria.

En esa línea, este estudio proyecta la posibilidad de medir nuevamente estas competencias descritas en un segundo momento, creando así una línea de trabajo con mayor profundidad para analizar tales aspectos en edades posteriores como podrían ser los 18 meses o los 24, con el fin de contribuir no sólo a identificar los mecanismos subyacentes de la comunicación, sino también para el diseño de intervenciones orientadas a optimizar las competencias de comunicación en las primeras fases del desarrollo temprano del infante.

\section{Conclusión}

Haciendo referencia a los resultados obtenidos, se puede apreciar como la atención conjunta guarda una estrecha relación con el desarrollo del lenguaje, siendo determinante en su correcta adquisición, además de ser un pilar fundamental en la detección de posibles patologías lingüísticas en los primeros años de vida de un niño.

De esta manera, se establece que la atención conjunta puede ser un factor protector para la correcta adquisición y desarrollo del lenguaje en la infancia. Todo ello es debido a la presencia de conductas y habilidades que favorecen la aparición de competencias prelingüísticas, siendo la atención conjunta un prerrequisito del lenguaje oral. Por otro lado, las conductas de atención conjunta y comunicación de los bebés aumentan de 8 meses a los 12 de manera significativa. Se muestra, pues, que los 12 meses es el punto crítico en donde surgen de manera exponencial las conductas e interacciones correspondientes a la atención conjunta del infante.

Sin embargo, es necesario destacar que, durante el primer año de vida, es realmente difícil el comprobar de forma exacta el lenguaje que posee el bebé debido a las pocas emisiones que produce. Las pruebas estandarizadas y los registros de conductas existentes en la actualidad son subjetivos, que pueden provocar sesgos en la codificación de las conductas prelingüísticas y lingüísticas del menor, por lo que es necesario que los evaluadores tengan una destacada experiencia y destreza en la evaluación del lenguaje en niños durante sus primeros años de vida.

\section{Referencia}

Caruana, N. L. (2015). The neural time course of evaluating self囚initiated joint attention bids. Brain Cogn., 98, 43-52.

Pfeiffer, U. J. (2014). Why we interact: on the functional role of the striatum in the subjective experience of social interaction. Neurolmage, 101, 124-137. Neurolmage(101), 124-137.

Yoo, K. \&.-S. (2013). Effects of negative temperament on 5-month-old infants' behaviorduring the still-face paradigm. Infant Behavior and Development, 36, 344-348. Infant Behavior and Development, 36, 344-348.

Olson, J. M. (2011). Infants' gestures influence mothers' provision of object, action and internal state labels. Journal of Child Language, 38(5), 1028-54.

Mundy, P. S. (2009). A Parallel and Distributed Processing Model of Joint Attention, Social-Cognition and Autism. Autism, 2(1), 2-21.

Mundy, P. K. (2016). Brief Report: Joint attention and information processing in children with higher functioning autism spectrum disorders. J. Autism Dev. Disord, 46-16.
Cameron-Faulkner, T. T. (2015). The relationship between infant holdout and gives, and pointing. I. Infancy(20), 576-58.

Mundy, P. (2016). Autism and Joint Attention: Developmental, Neuroscience and Clinical Fundamentals. New York: Guilford Publications Inc. Neuroscience and Clinical Fundamentals. New York: Guilford Publications Inc.

Cochet, H. J. (2016). Relationships between declarative pointing and theory of mind abilities in 3-4 year-olds. European Journal of Developmental Psychology, 14, 14, 1-13.

Matthews, D. B. (2012). Origins of the human pointing gesture: a training study. Developmental science, 15(6), 817-829. Developmental science, 15(6), 817-829.

Kristen, S. S. (2011). Infants' Joint Attention Skills Predict Toddlers' Emerging Mental State Language. Developmental Psychology, 47(5), 120-7.

Bruner, J. (1983). Child's talk: Learning to use language. . England: Oxford.

Butterworth, G. E. (1991). The ontogeny and phylogeny of joint visual attention. . En Butterworth, G. E. (1991). The ontogeny and phylogeny of joint visual attention. England: Oxford. England: Oxford.

Sodian, B. \&.-A. (2015). Declarative joint attention as a foundation of theory of mind. Developmental psychology, 51(9), 1190.

Ramenzoni,V.C.(2016). Thesocial reach:8-month-olds reachforunobtainable objects in the presence of another person. Psychological Science, 27, 1278-1285.

Cano Villagrasa, A. \&. (2020). Estrategias de afrontamiento del estrés en familias compuestas por niños con Trastorno del Neurodesarrollo. La revista d'atenciò precoç, 40, 1-15. Desenvolupa, La revista d'atenciò precoç, (40), 1-15.

Sorondo, B. M.-S. (2015). Associations between infant temperament, maternal stress, and infants' sleep across the first year of life. I. Infant Behavior and Development(39), 131-135.

Kuhn, L. J.-F.-1. (2014). Early communicative gestures prospectively predict language development and executive function in early childhood. Child Development, 85, 1898-1914. Child Development(85), 1898-1914.

Acosta, V. M. (2013). Análisis de las dificultades en el discurso narrativo en alumnado con Trastorno Específico del Lenguaje. Revista de Logopedia, Foniatría y Audiología, 33(4), 165-171.

Acosta, V. D. (2016). Uso de las narraciones para la evaluación y la intervención en el Trastorno Específico del Lenguaje (TEL)En M. Martín y R. López ( Coords). Claves de la logopedia en el siglo XXI, 337-354.

Maldonado, J. B. (2013). Variabilidad morfosintáctica en las narrativas de niños con trastorno específico de lenguaje. En R Barriga (ed) Las narrativas y su impacto en el desarrollo lingüistico infantil. México, D.F.: El Colegio de México.

Laurence B, L. (2014). Children with Specific Language Impairment. Cambridge: MIT Press.

Mundy, P. D. (2003). A manual for the abridged Early Social Communication Scales (ESCS). Florida: Coral Gables.

López-Ornat, S. G. (2005). Inventario de Desarrollo Comunicativo MacArthur. Madrid: TEA Ediciones.

Elgier, A. M. (2017). Seguimiento del gesto de señalar y de la mirada en estadios tempranos del desarrollo. Apuntes de Ciencia \& Sociedad, 7(1), 11-20.

Gutiérrez-Ruiz, K. (2019). Características tempranas y predictores de la severidad del cuadro clínico en el trastorno del espectro autista. 12(2), $12-25$. 